\title{
CSO AND CARMA OBSERVATIONS OF L1157. I. A DEEP SEARCH FOR HYDROXYLAMINE $\left(\mathrm{NH}_{2} \mathrm{OH}\right)$
}

\author{
Brett A. McGuire ${ }^{1,2,7}$, P. Brandon Carroll ${ }^{2}$, Niklaus M. Dollhopf ${ }^{1,3}$, Nathan R. Crockett ${ }^{4}$, Joanna F. Corby ${ }^{1,3}$, \\ Ryan A. Loomis ${ }^{5}$, Andrew M. Burkhardt ${ }^{1,3}$, Christopher Shingledecker ${ }^{6}$, Geoffrey A. Blake ${ }^{2,4}$, and \\ AnTHONY J. REMIJAN ${ }^{1}$ \\ ${ }^{1}$ National Radio Astronomy Observatory, Charlottesville, VA 22903, USA \\ ${ }^{2}$ Division of Chemistry and Chemical Engineering, California Institute of Technology, Pasadena, CA 91125, USA \\ ${ }^{3}$ Department of Astronomy, University of Virginia, Charlottesville, VA 22903, USA \\ ${ }^{4}$ Division of Geological and Planetary Sciences, California Institute of Technology, Pasadena, CA 91125, USA \\ ${ }^{5}$ Department of Astronomy, Harvard University, Cambridge, MA 02138, USA \\ ${ }^{6}$ Department of Chemistry, University of Virginia, Charlottesville, VA 22903, USA \\ Received 2015 April 13; accepted 2015 September 11; published 2015 October 9
}

\begin{abstract}
A deep search for the potential glycine precursor hydroxylamine $\left(\mathrm{NH}_{2} \mathrm{OH}\right)$ using the Caltech Submillimeter Observatory (CSO) at $\lambda=1.3 \mathrm{~mm}$ and the Combined Array for Research in Millimeter-wave Astronomy at $\lambda=3 \mathrm{~mm}$ is presented toward the molecular outflow L1157, targeting the B1 and B2 shocked regions. We report non-detections of $\mathrm{NH}_{2} \mathrm{OH}$ in both sources. We perform a non-LTE analysis of $\mathrm{CH}_{3} \mathrm{OH}$ observed in our CSO spectra to derive the kinetic temperatures and densities in the shocked regions. Using these parameters, we derive upper limit column densities of $\mathrm{NH}_{2} \mathrm{OH}$ of $\leqslant 1.4 \times 10^{13} \mathrm{~cm}^{-2}$ and $\leqslant 1.5 \times 10^{13} \mathrm{~cm}^{-2}$ toward the B1 and B2 shocks, respectively, and upper limit relative abundances of $N_{\mathrm{NH}_{2} \mathrm{OH}} / N_{\mathrm{H}_{2}} \leqslant 1.4 \times 10^{-8}$ and $\leqslant 1.5 \times 10^{-8}$, respectively.
\end{abstract}

Key words: astrochemistry - ISM: individual objects (L1157) - ISM: molecules

\section{INTRODUCTION}

Glycine $\left(\mathrm{NH}_{2} \mathrm{CH}_{2} \mathrm{COOH}\right)$, the simplest amino acid, has been identified in cometary (Elsila et al. 2009) and meteoritic samples (Kvenvolden et al. 1970); however, it is not yet known whether the species is formed in these solid bodies, in the interstellar medium (ISM), or both. Indeed, a detection in the ISM has so far proven elusive (Snyder et al. 2005; Cunningham et al. 2007; Jones et al. 2007). Although chemical models predict the formation of glycine in the ISM in low abundance (see, e.g., Garrod 2013 and the references therein), it has been difficult to observationally constrain them without a detection of the species. The relative likelihood of different possible formation routes can, however, be constrained by careful observation of the reactants used in the chemical models to form glycine.

One pathway that has garnered significant interest is the formation of glycine through the reaction of hydroxylamine $\left(\mathrm{NH}_{2} \mathrm{OH}\right)$, or its protonated and ionized derivatives, with acetic acid $\left(\mathrm{CH}_{3} \mathrm{COOH}\right)$, a known interstellar molecule (Mehringer et al. 1997). Ionization or protonation of $\mathrm{NH}_{2} \mathrm{OH}$ under interstellar conditions should be efficient (Angelelli et al. 1995; Boulet et al. 1999), and subsequent laboratory work has demonstrated the formation of glycine from $\mathrm{NH}_{2} \mathrm{OH}$ and its ionized and protonated forms (Blagojevic et al. 2003; Snow et al. 2007). Although recent theoretical work has suggested that gas-phase routes forming glycine through these reactions are inefficient under interstellar conditions (Barrientos et al. 2012), condensed-phase surface-mediated reactions remain a possibility, and observational constraints for the precursors are still desirable for the refinement of models. While acetic acid is readily constrained, $\mathrm{NH}_{2} \mathrm{OH}$ has yet to be observed in the ISM.

\footnotetext{
${ }^{7}$ Jansky Fellow of the National Radio Astronomy Observatory.
}

In recent years, laboratory work has shown that a number of pathways exist which result in the efficient formation of $\mathrm{NH}_{2} \mathrm{OH}$ on grain surfaces. Zheng \& Kaiser (2010) demonstrated the formation of $\mathrm{NH}_{2} \mathrm{OH}$ in $\mathrm{H}_{2} \mathrm{O}-\mathrm{NH}_{3}$ ices after UVirradiation. Formation by successive hydrogenation of $\mathrm{NO}$ has been shown to proceed efficiently, without barriers, and in high yield (Congiu et al. 2012a, 2012b; Fedoseev et al. 2012; Ioppolo et al. 2014; Minissale et al. 2014). Most recently, He et al. (2015) demonstrated the efficient formation of $\mathrm{NH}_{2} \mathrm{OH}$ via the oxidation of $\mathrm{NH}_{3}$ in ices with a low barrier to activation (of the order of $1000 \mathrm{~K}$ ).

Earlier modeling work by Garrod et al. (2008) also suggested that $\mathrm{NH}_{2} \mathrm{OH}$ is formed in high abundance in interstellar ices, and is subsequently liberated into the gas phase during the warm-up period of emerging hot cores and hot corinos in readily-detectable quantities. Observational efforts by Pulliam et al. (2012), however, failed to detect $\mathrm{NH}_{2} \mathrm{OH}$ toward a selection of such sources known to be rich in complex organic material (e.g., Sgr B2(N) and Orion-KL), establishing upper limits of $N_{\mathrm{NH}_{2} \mathrm{OH}} / N_{\mathrm{H}_{2}}<10^{-9}-10^{-11}$. Refinement of the Garrod et al. (2008) model in subsequent work (Garrod 2013), as well as limited modeling presented in the aforementioned laboratory studies, resulted in predicted gas-phase abundances in agreement with the upper limits established by Pulliam et al. (2012). Yet, with the inclusion of the $\mathrm{H}+\mathrm{HNO} \rightarrow \mathrm{HNOH}$ pathway in the model, as described by the laboratory work of Congiu et al. (2012a), condensed-phase abundances of $\mathrm{NH}_{2} \mathrm{OH}$ again approach $N_{\mathrm{NH}_{2} \mathrm{OH}} / N_{\mathrm{H}_{2}} \sim 10^{-6}$, with gas-phase abundances of $N_{\mathrm{NH}_{2} \mathrm{OH}} / N_{\mathrm{H}_{2}} \sim 10^{-7}$.

The Garrod (2013) model largely assumes that the release of $\mathrm{NH}_{2} \mathrm{OH}$ into the gas phase is a gradual process dominated by the warm-up of the hot core. Both the laboratory work and the models, however, predict that $\mathrm{NH}_{2} \mathrm{OH}$ is initially formed in large abundance at very cold $(<20 \mathrm{~K})$ temperatures and early in the evolution of these sources. Thus, the most optimistic source for a detection of gas-phase $\mathrm{NH}_{2} \mathrm{OH}$ is one where the reservoir 
of condensed-phase $\mathrm{NH}_{2} \mathrm{OH}$ formed at low temperatures is liberated en masse into the gas phase prior to its release by thermal mechanisms.

Shocked regions displaying high degrees of molecular complexity likely represent this best-case scenario. In these regions, complex molecules are formed efficiently in ices at low temperatures, but are not otherwise liberated into the gas phase except by thermal desorption at much greater temperatures. When these ices are subjected to shocks, however, the mantle is non-thermally ejected into the gas phase, resulting in large abundances of relatively cool $\left(T_{\text {rot }}<100 \mathrm{~K}\right)$, complex organic material (see, e.g., Requena-Torres et al. 2006). One of the most prominent of these regions is the young protostellar outflow L1157. Numerous recent studies report high degrees of molecular complexity arising from shocked regions within the outflow, which originates in cold, quiescent gas around the protostar (see, e.g., Arce et al. 2008 and Codella et al. 2015).

Here, we present deep searches for $\mathrm{NH}_{2} \mathrm{OH}$ using the Caltech Submillimeter Observatory (CSO) at $\lambda=1.3 \mathrm{~mm}$ and the Combined Array for Research in Millimeter-wave Astronomy (CARMA) at $\lambda=3 \mathrm{~mm}$ toward the L1157 outflow. We report non-detections of $\mathrm{NH}_{2} \mathrm{OH}$ in both searches. In order to derive upper limits to the column density of $\mathrm{NH}_{2} \mathrm{OH}$, we use transitions of $\mathrm{CH}_{3} \mathrm{OH}$ observed with the $\mathrm{CSO}$ to constrain the kinetic temperature and density in the shocked gas using a radiative-transfer approach, and CARMA images of $\mathrm{CH}_{3} \mathrm{OH}$ and $\mathrm{HNCO}$ to determine the size of the shocked gas. Finally, we estimate upper limits to the abundance of $\mathrm{NH}_{2} \mathrm{OH}$ and discuss possible implications.

\section{L1157}

L1157 is a dark cloud in Cepheus located at a distance of $\sim 250$ pc (Looney et al. 2007) which contains a prototypical shocked bipolar outflow from a Class 0 protostar. It has been the subject of great interest in the last 20 years, with numerous studies investigating the physical conditions within the source. Due to the variety of methods used in these studies, direct comparisons between their results are challenging. Nevertheless, an overall picture does arise, and this general description will be sufficient for the discussion presented here.

Originating in the cold, quiescent gas $(T \sim 13 \mathrm{~K}$, Bachiller et al. 1993) surrounding the Class 0 protostar L1157-mm, the southern lobe of the accelerated outflow $(T \sim 50-100 \mathrm{~K}$; Bachiller et al. 1993) has undergone two major shocks, referred to as L1157-B1 and L1157-B2. The B1 shock is younger and warmer $\left(\sim 2000\right.$ years, $\left.T_{\text {kin }} \sim 80-100 \mathrm{~K}\right)$ than the B2 shock ( $\sim 4000$ years, $\left.T_{\text {kin }} \sim 20-60 \mathrm{~K}\right)$, and many complex chemical species are observed in enhanced abundance toward both shocks due to non-thermal desorption from grains (Mendoza et al. 2014; Codella et al. 2015). While the absolute values of these physical parameters vary somewhat within the literature, this qualitative picture and enhancement in chemical abundance is consistently reported.

\section{CSO OBSERVATIONS}

The spectrum toward L1157 obtained with the CSO was collected over eight nights in 2012 July-September, and seven nights in 2014 September and December. The telescope was pointed at the B1 shocked region at $\alpha(\mathrm{J} 2000)=20^{\mathrm{h}} 39^{\mathrm{m}} 07^{\mathrm{s}} .7, \delta$ $(\mathrm{J} 2000)=68^{\circ} 01^{\prime} 15^{\prime \prime} .5$ and the $\mathrm{B} 2$ shocked region at $\alpha$ $(\mathrm{J} 2000)=20^{\mathrm{h}} 39^{\mathrm{m}} 13^{\mathrm{s}}, \delta(\mathrm{J} 2000)=68^{\circ} 00^{\prime} 37^{\prime \prime}($ see Figure 1$)$, and

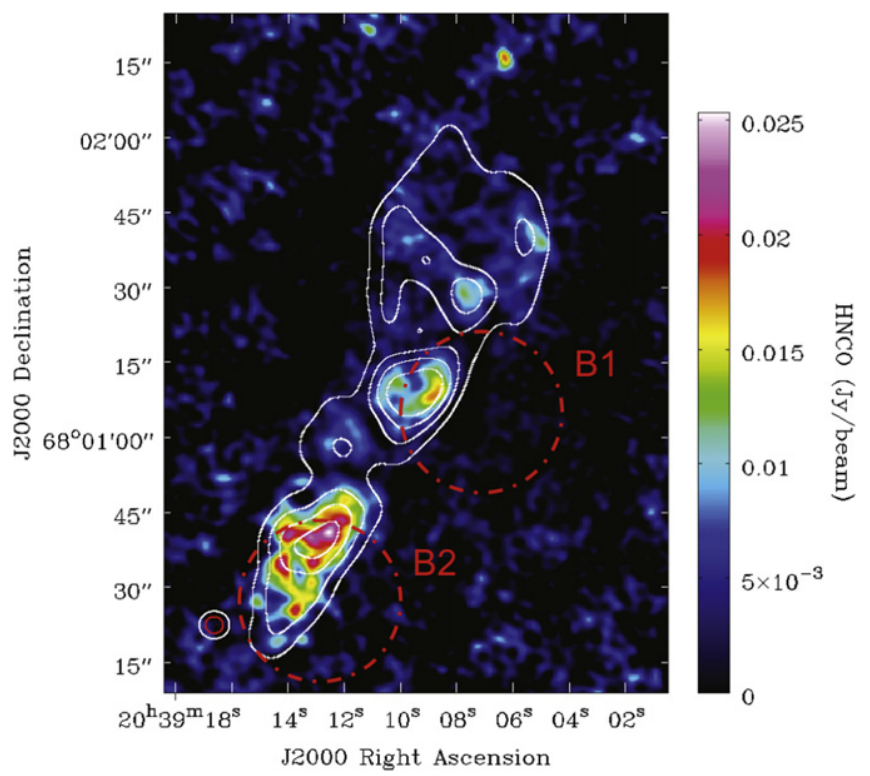

Figure 1. CARMA observations of $\mathrm{CH}_{3} \mathrm{OH}$ and $\mathrm{HNCO}$ toward L1157. Contours are an integrated Moment 0 map of the $2_{-1,2}-1_{-1,1}, 2_{0,2}-1_{0,1}++$, and $2_{0,2}-1_{0,1}$ transitions $\left(E_{u}=12.5 \mathrm{~K}, 7 \mathrm{~K}\right.$, and $20.1 \mathrm{~K}$, respectively) of $\mathrm{CH}_{3} \mathrm{OH}$ at contour levels of $0.16,0.32,0.49,0.66$, and $0.82 \mathrm{Jy}_{\text {beam }}{ }^{-1}$. These are overlaid on an integrated Moment 0 map of the $4_{0,4}-3_{0,3}$ transition of HNCO spanning 0 $\rightarrow 0.025 \mathrm{Jy}$ beam $^{-1}$ in a single color cycle. The HNCO transitions trace the warmer, compact shocks, while the $\mathrm{CH}_{3} \mathrm{OH}$ transitions reveal the overall structure of the colder, diffuse outflow. The location of the two targeted observations of B1 and B2 with the CSO are shown as dashed white lines, approximately equal to the CSO beam size $\left(\sim 30^{\prime \prime}\right)$ at the observed frequencies.

spectra were adjusted to a $v_{\mathrm{LSR}}=1.75 \mathrm{~km} \mathrm{~s}^{-1}$. A small subset of scans toward B1 were obtained at a second position offset by $-4^{\prime \prime}$ in declination. The CSO $230 / 460 \mathrm{GHz}$ sidecab doublesideband (DSB) heterodyne receiver was used in fair weather $\left(\tau_{220}=0.1-0.25\right)$ resulting in typical system temperatures of $300-500 \mathrm{~K}$. The backend was a fast Fourier-transform spectrometer which provided $1 \mathrm{GHz}$ of DSB data at $122 \mathrm{kHz}$ resolution $\left(\sim 0.2 \mathrm{~km} \mathrm{~s}^{-1}\right.$ at $\left.230 \mathrm{GHz}\right)$. The total frequency coverage was 188-193, 200-205, 237-243, and 249-255 GHz toward B1, and $237-243$ and $249-255 \mathrm{GHz}$ toward B2. The targeted transitions of $\mathrm{NH}_{2} \mathrm{OH}$ are given in Table 1 .

A chopping secondary mirror with a throw of $4^{\prime}$ was used for ON-OFF calibration. Pointing was performed every $\sim 2 \mathrm{hr}$ and typically converged to within $1^{\prime \prime}-2^{\prime \prime}$. The raw data were calibrated using the standard chopper wheel calibration method resulting in intensities on the atmosphere-corrected $T_{\mathrm{A}}^{*}$ temperature scale. These were then corrected to the main beam temperature scale, $T_{\mathrm{mb}}$, where $T_{\mathrm{mb}}=T_{\mathrm{A}}^{*} / \eta_{\mathrm{mb}}$. For these observations, the main beam efficiency was $\eta_{\mathrm{b}}=0.70$. The spectra were collected in DSB mode at a variety of IF settings to allow for a robust deconvolution. The CLASS package from the GILDAS suite of programs ${ }^{7}$ was used for the data reduction. Spurious signals were removed from the spectra, which were then baseline subtracted using a polynomial fit. The standard CLASS deconvolution routine was used to generate single-sideband data. The spectra were then Hanning smoothed to a resolution of $\sim 1.4 \mathrm{~km} \mathrm{~s}^{-1}$. The average FWHM linewidth in B1 was $\sim 7.9 \mathrm{~km} \mathrm{~s}^{-1}$, and in $\mathrm{B} 2$ was $\sim 5.2 \mathrm{~km} \mathrm{~s}^{-1}$.

\footnotetext{
Institut de Radioastronomie Millimétrique, Grenoble, France-http://www iram.fr/IRAMFR/GILDAS
} 
Table 1

Targeted $\mathrm{NH}_{2} \mathrm{OH}$ Transitions, Spectroscopic Parameters, Assumed Collisional Coefficients, and Critical Densities

\begin{tabular}{|c|c|c|c|c|c|c|}
\hline Transition & $\begin{array}{l}\text { Frequency }^{\mathrm{a}} \\
(\mathrm{MHz})\end{array}$ & $\begin{array}{l}E_{U} \\
(\mathrm{~K})\end{array}$ & $\begin{array}{c}S_{i j} \mu^{2} \\
\left(\text { Debye }^{2}\right)\end{array}$ & $\begin{array}{c}\gamma^{\mathrm{b}} \\
\left(\mathrm{cm}^{3} \mathrm{~s}^{-1}\right)\end{array}$ & $\begin{array}{c}\log (A) \\
\left(\log \left(\mathrm{s}^{-1}\right)\right)\end{array}$ & $\begin{array}{c}n_{\mathrm{cr}}{ }^{\mathrm{c}} \\
\left(\mathrm{cm}^{-3}\right)\end{array}$ \\
\hline $2_{1,2}-1_{1,1}$ & $100683.58(20)$ & 15.204 & 0.520 & $4.1 \times 10^{-11}$ & -5.9079 & $3 \times 10^{4}$ \\
\hline $2_{0,2}-1_{0,1}$ & $100748.23(20)$ & 7.2527 & 0.694 & $5.5 \times 10^{-11}$ & -5.7821 & $3 \times 10^{4}$ \\
\hline $5_{1,5}-4_{1,4}$ & $251677.3666(78)$ & 44.194 & 1.665 & $4.0 \times 10^{-12}$ & -4.5515 & $7 \times 10^{6}$ \\
\hline $5_{4,1}-4_{4,0}$ & $251734.8061(82)$ & 163.51 & 0.625 & $2.4 \times 10^{-11}$ & -4.9771 & $4 \times 10^{5}$ \\
\hline $5_{4,2}-4_{4,1}$ & $251734.8061(82)$ & 163.51 & 0.625 & $2.4 \times 10^{-11}$ & -4.9771 & $4 \times 10^{5}$ \\
\hline $5_{2,4}-4_{2,3}$ & $251811.9913(70)$ & 68.081 & 1.457 & $2.0 \times 10^{-13}$ & -4.6088 & $1 \times 10^{8}$ \\
\hline $5_{2,3}-4_{2,2}$ & $251813.8609(70)$ & 68.081 & 1.457 & $2.0 \times 10^{-13}$ & -4.6087 & $1 \times 10^{8}$ \\
\hline $5_{0,5}-4_{0,4}$ & 251838.4937(79) & 36.261 & 1.735 & $1.2 \times 10^{-10}$ & -4.5329 & $1 \times 10^{5}$ \\
\hline $5_{1,4}-4_{1,3}$ & $251987.1350(78)$ & 44.239 & 1.665 & $4.0 \times 10^{-12}$ & -4.5498 & $1 \times 10^{6}$ \\
\hline
\end{tabular}

Notes.

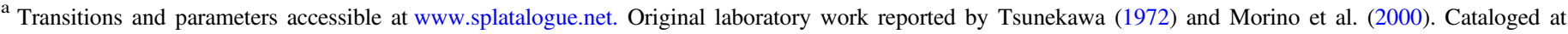
CDMS (Müller et al. 2005).

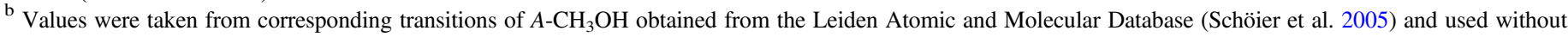
further modification. Original data reported by Rabli \& Flower (2010).

${ }^{\mathrm{c}}$ Given as $n_{\mathrm{cr}}=A / \gamma$.

\section{CARMA OBSERVATIONS}

A total of $89.3 \mathrm{hr}$ of observations were conducted with the CARMA 15-element array in C-configuration (2013 May), D-configuration (2012 October, November), and E-configuration (2012 August) at $\lambda=3 \mathrm{~mm}$. The phase center for these observations was $\alpha(\mathrm{J} 2000)=20^{\mathrm{h}} 39^{\mathrm{m}} 07^{\mathrm{s}} .7, \delta$ $(\mathrm{J} 2000)=68^{\circ} 01^{\prime} 11^{\prime \prime} 5$. The CARMA correlator was used in its $62 \mathrm{MHz}$ bandwidth, 3-bit mode providing 255 channels across the band for a resolution of $243 \mathrm{kHz}$ or $0.7 \mathrm{~km} \mathrm{~s}^{-1}$. The three targeted transitions of $\mathrm{NH}_{2} \mathrm{OH}$ are given in Table 1.

MWC349 and Neptune were used as primary flux calibrators; the passband calibrators were $1635+381,2232+117$, $0102+584, \quad 1743-038,2015+372$, and $3 \mathrm{C} 84$. The gain calibrator was $1927+739$. Data reduction was completed using the Miriad package. Data were flagged for antennas that were offline or malfunctioning during observation or when the phases in the calibrators had deviations greater than 30 degrees from a smooth trend line. Image cleaning was also performed using Miriad. The robust factor tended toward natural weighting with cell size set to 0". 4 and image size of 1024 pixels. No channel averaging was applied for the maps shown; however, channel averaging to $1.4 \mathrm{~km} \mathrm{~s}^{-1}$ was used on windows targeting hydroxylamine transitions. Clean regions were drawn with the polygon tool around clear emission and cleaned to an average noise of $0.7 \mathrm{mJy}$ beam $^{-1}$. The restoring beam was typically $\sim 3$ "! $4 \times 3$ " 2 .

The full set of observations toward L1157 CARMA included a host of other molecular species, and will be presented in a follow-up paper by N. M. Dollhopf et al. (2015, in preparation).

\section{NON-LTE MODELING OF $\mathrm{CH}_{3} \mathrm{OH}$}

An accurate determination of $\mathrm{NH}_{2} \mathrm{OH}$ column density upper limits in the $\mathrm{B} 1$ and $\mathrm{B} 2$ shocks requires the kinetic temperature and density in these regions. To obtain these, we fit the observed $\mathrm{CH}_{3} \mathrm{OH}$ emission in the same spectral window (Table 2) to a non-LTE model of the outflow and shock, based on a physical model derived from our CARMA observations using the RADEX code (van der Tak et al. 2007).

First, we determined the approximate spatial extent and location of the shocked gas from the $\mathrm{CH}_{3} \mathrm{OH}$ emission observed with CARMA. Figure 1 shows the CSO beam at the targeted positions overlaid on our CARMA observations of $\mathrm{CH}_{3} \mathrm{OH}$ and $\mathrm{HNCO}^{8}$ at $\lambda=3 \mathrm{~mm}$. The pointing position for B1 was chosen from the literature prior to our CARMA observations, and is roughly centered on the shock front rather than the peak of the shock emission. The pointing position for B2 was chosen after we had acquired the CARMA observations, and is centered on the peak of the shocked HNCO emission. In both cases, the shock is nearly completely contained within the beam, and we estimate a reasonable equivalent size of $\sim 12^{\prime \prime}$ for the shock, and $\sim 20^{\prime \prime}$ for the second component, within our $\sim 30^{\prime \prime}$ CSO beam. In B1, the shock is not centered in the beam, and thus any emission will suffer more significant fall-off effects than in B2.

We made the initial assumption that the two components in our fit represented the shocked gas and the outflow. Under these assumptions, and based on our CARMA observation as well as prior studies (Bachiller et al. 1995), we constrained the fit of the $\mathrm{CH}_{3} \mathrm{OH}$ emission with the following assumptions.

1. The kinetic temperature of the shock was no less than that of the outflow.

2. The density of the shock was no less than that of the outflow.

3. The column density of $\mathrm{CH}_{3} \mathrm{OH}$ was no less in the shock than in the outflow.

Assumptions (1) and (2) are well-substantiated in the literature, both toward L1157 (Bachiller et al. 1995) and in shocked environments in general (van Dishoeck \& Blake 1998), and assumption (3) agrees with the previous findings of Bachiller et al. (1995). Using these constraints, and the twosource component model described earlier, we performed a reduced- $\chi^{2}$ analysis of a grid of models for the CSO

\footnotetext{
8 Details and analysis of the HNCO observations will be presented in Paper II by N. M. Dollhopf et al. (2015, in preparation).
} 
Table 2

Observed $\mathrm{CH}_{3} \mathrm{OH}$ Transitions, Spectroscopic Parameters, Collisional Coefficients, and Critical Densities

\begin{tabular}{|c|c|c|c|c|c|c|}
\hline Transition $^{\mathrm{a}}$ & $\begin{array}{c}\text { Frequency } \\
(\mathrm{MHz})\end{array}$ & $\begin{array}{l}E_{U} \\
(\mathrm{~K})\end{array}$ & $\begin{array}{c}S_{i j} \mu^{2} \\
\left(\text { Debye }^{2}\right)\end{array}$ & $\begin{array}{c}\gamma^{\mathrm{c}} \\
\left(\mathrm{cm}^{3} \mathrm{~s}^{-1}\right)\end{array}$ & $\begin{array}{c}\log (A) \\
\left(\log \left(\mathrm{s}^{-1}\right)\right)\end{array}$ & $\begin{array}{c}n_{\mathrm{cr}}{ }^{\mathrm{d}} \\
\left(\mathrm{cm}^{-3}\right)\end{array}$ \\
\hline \multicolumn{7}{|c|}{$A-\mathrm{CH}_{3} \mathrm{OH}$} \\
\hline $2_{0,2}-1_{0,1}++$ & $96741.375(5)$ & 6.9650 & 1.617 & $5.5 \times 10^{-11}$ & -5.4675 & $6 \times 10^{4}$ \\
\hline $5_{1,5}-4_{1,4}++$ & 239746.253 & 49.059 & 3.885 & $4.3 \times 10^{-12}$ & -4.2468 & $1 \times 10^{7}$ \\
\hline $5_{0,5}-4_{0,4}++$ & 241791.431 & 34.817 & 4.043 & $1.2 \times 10^{-10}$ & -4.2184 & $5 \times 10^{5}$ \\
\hline $5_{3,3}-4_{3,2}++$ & $241832.91(20)$ & 84.618 & 2.578 & $1.6 \times 10^{-11}$ & -4.4137 & $2 \times 10^{6}$ \\
\hline $5_{3,2}-4_{3,1}--$ & $241832.91(20)$ & 84.618 & 2.578 & $4.6 \times 10^{-11}$ & -4.4137 & $8 \times 10^{5}$ \\
\hline $5_{2,4}-4_{2,3}--$ & 241842.324 & 72.530 & 3.415 & $1.3 \times 10^{-13}$ & -4.2915 & $4 \times 10^{8}$ \\
\hline $5_{2,3}-4_{2,2}++$ & 241887.704 & 72.533 & 3.415 & $1.8 \times 10^{-13}$ & -4.2912 & $3 \times 10^{8}$ \\
\hline $5_{1,4}-4_{1,3}--$ & 243915.826 & 49.661 & 3.885 & $3.8 \times 10^{-13}$ & -4.2243 & $2 \times 10^{8}$ \\
\hline $11_{0,11}-10_{1,10}++$ & 250506.980 & 153.10 & 10.63 & $9.0 \times 10^{-13}$ & -4.0728 & $9 \times 10^{7}$ \\
\hline $8_{3,5}-8_{2,6}-+$ & 251517.262 & 133.36 & 7.308 & $8.1 \times 10^{-13}$ & -4.0990 & $1 \times 10^{8}$ \\
\hline $7_{3,4}-7_{2,5}-+$ & 251641.667 & 114.79 & 6.279 & $1.4 \times 10^{-12}$ & -4.1099 & $6 \times 10^{7}$ \\
\hline $6_{3,3}-6_{2,4}-+$ & 251738.520 & 98.546 & 5.224 & $6.7 \times 10^{-12}$ & -4.1272 & $1 \times 10^{7}$ \\
\hline $5_{3,2}-5_{2,3}-+$ & 251811.882 & 84.618 & 4.126 & $1.8 \times 10^{-11}$ & -4.1567 & $4 \times 10^{6}$ \\
\hline $4_{3,1}-4_{2,2}-+$ & 251866.579 & 73.012 & 2.954 & $2.2 \times 10^{-11}$ & -4.2144 & $3 \times 10^{6}$ \\
\hline $5_{3,3}-5_{2,4}+-$ & 251890.901 & 84.619 & 4.125 & $8.1 \times 10^{-12}$ & -4.1564 & $9 \times 10^{6}$ \\
\hline $6_{3,4}-6_{2,5}+-$ & 251895.728 & 98.545 & 5.220 & $2.6 \times 10^{-12}$ & -4.1266 & $3 \times 10^{7}$ \\
\hline $4_{3,2}-4_{2,3}+-$ & 251900.495 & 73.012 & 2.953 & $4.5 \times 10^{-11}$ & -4.2143 & $1 \times 10^{6}$ \\
\hline $3_{3,0}-3_{2,1}-+$ & 251905.812 & 63.727 & 1.641 & $3.1 \times 10^{-11}$ & -4.3603 & $1 \times 10^{6}$ \\
\hline $3_{3,1}-3_{2,2}+-$ & 251917.042 & 63.727 & 1.641 & $1.3 \times 10^{-12}$ & -4.3603 & $3 \times 10^{7}$ \\
\hline $7_{3,5}-7_{2,6}+-$ & 251923.631 & 114.79 & 6.272 & $8.5 \times 10^{-11}$ & -4.1089 & $9 \times 10^{5}$ \\
\hline $8_{3,6}-8_{2,7}+-$ & 251984.702 & 133.36 & 7.293 & $2.1 \times 10^{-11}$ & -4.0975 & $4 \times 10^{6}$ \\
\hline \multicolumn{7}{|c|}{$E-\mathrm{CH}_{3} \mathrm{OH}$} \\
\hline $2_{-1,2}-1_{-1,2}$ & $96739.362(5)$ & 12.541 & 1.213 & $1.4 \times 10^{-13}$ & -5.5922 & $2 \times 10^{7}$ \\
\hline $2_{0,2}-1_{0,2}$ & $96744.550(5)$ & 20.090 & 1.617 & $9.5 \times 10^{-12}$ & -5.4675 & $4 \times 10^{5}$ \\
\hline $2_{1,1}-1_{1,0}$ & $96755.511(5)$ & 28.011 & 1.244 & $3.7 \times 10^{-11}$ & -5.5811 & $7 \times 10^{4}$ \\
\hline $5_{0,5}-4_{0,4}$ & 241700.219 & 47.934 & 4.040 & $3.0 \times 10^{-11}$ & -4.2192 & $2 \times 10^{6}$ \\
\hline $5_{-1,5}-4_{-1,4}$ & 241767.224 & 40.391 & 3.882 & $7.9 \times 10^{-13}$ & -4.2362 & $7 \times 10^{7}$ \\
\hline $5_{3,2}-4_{3,1}$ & 241843.646 & 82.531 & 2.587 & $1.5 \times 10^{-12}$ & -4.4120 & $3 \times 10^{7}$ \\
\hline $5_{1,4}-4_{1,3}$ & 241879.073 & 55.871 & 3.980 & $3.1 \times 10^{-12}$ & -4.2248 & $2 \times 10^{7}$ \\
\hline $5_{-2,4}-4-2,3$ & 241904.152 & 60.725 & 3.399 & $1.1 \times 10^{-13}$ & -4.2932 & $5 \times 10^{8}$ \\
\hline $5_{2,3}-4_{2,2}$ & 241904.645 & 57.069 & 3.356 & $2.5 \times 10^{-12}$ & -4.2987 & $2 \times 10^{7}$ \\
\hline $20,2-1_{-1,1}$ & 254015.340 & 20.090 & 0.499 & $4.7 \times 10^{-13}$ & -4.7208 & $4 \times 10^{7}$ \\
\hline
\end{tabular}

Notes. Only those transitions with a modeled peak intensity above $5 \mathrm{mK}$ are listed here. A complete modeled spectrum is available as supplementary information. a "+" and "-" refer to $A+$ and $A-$ parity states, respectively. For $E-\mathrm{CH}_{3} \mathrm{OH}$, a negative value of $K_{a}$ is used to differentiate between $(+) E_{1}$ and $(-) E_{2}$ states, which belong to the same $E$ symmetry species.

${ }^{\mathrm{b}}$ Transitions and parameters accessible at www.splatalogue.net. Original laboratory work reported by Xu \& Lovas (1997) and the references therein and by Müller et al. (2004). Cataloged at CDMS (Müller et al. 2005). Except where noted, uncertainties are $50 \mathrm{kHz}$.

${ }^{c}$ Values obtained from the Leiden Atomic and Molecular Database (Schöier et al. 2005). Original data reported by Rabli \& Flower (2010).

${ }^{\mathrm{d}}$ Given as $n_{\mathrm{cr}}=A / \gamma$.

Table 3

Best-fit Parameters for Two Components of $\mathrm{CH}_{3} \mathrm{OH}$ toward L1157-B1 and B2 Determined from Non-LTE RADEX Calculations

\begin{tabular}{|c|c|c|c|c|}
\hline \multirow[b]{2}{*}{ Parameter } & \multicolumn{2}{|c|}{ L1157-B1 } & \multicolumn{2}{|c|}{ L1157-B2 } \\
\hline & Component 1 & Component 2 & Component 1 & Component 2 \\
\hline$n_{\mathrm{H}_{2}}\left(\mathrm{~cm}^{-3}\right)$ & $3(1) \times 10^{5}$ & $3(1) \times 10^{5}$ & $6(2) \times 10^{5}$ & $6(2) \times 10^{5}$ \\
\hline$T_{\text {kin }}(\mathrm{K})$ & $60(20)$ & $10(3)$ & $50(15)$ & $10(3)$ \\
\hline$N_{\mathrm{CH}_{3} \mathrm{OH}}\left(\mathrm{cm}^{-2}\right)$ & $3(1) \times 10^{15}$ & $5(2) \times 10^{14}$ & $3(1) \times 10^{15}$ & $6(2) \times 10^{14}$ \\
\hline
\end{tabular}

Note. Errors given are from Equation (1) and calculated to be $\sim 32 \%$.

observations, simultaneously fitting $A$ - and $E-\mathrm{CH}_{3} \mathrm{OH}$ following the methods described in Crockett et al. (2014). A thorough discussion of the uncertainties in the fitting method is provided in the Appendix. 
Based on these uncertainties, and the assumptions described above, we find that the parameters in Table 3 represent a bestfit to the data, with reduced- $\chi^{2}$ values of 2.50 and 1.77 for B1 and B2, respectively. These values are likely due to the simplicity of our model relative to the complexity of the source. Literature sources (see, e.g., Lefloch et al. 2012) suggest that a structure with three or more components is more realistic, but such additional components would not be well-constrained in our case due to the limited number of observed $\mathrm{CH}_{3} \mathrm{OH}$ transitions. Additionally, there are likely quite large temperature and density gradients, especially in the newer, more compact B1 shock, which will contribute an additional error to the fit that is not accounted for in our analysis. Finally, $\mathrm{CH}_{3} \mathrm{OH}$ excitation is sensitive to the far-IR radiation field present, which we have assumed as the standard galactic background, any deviation from which would further impact the accuracy of the model and increased the ultimate $\chi^{2}$.

Simulated non-LTE spectra of $\mathrm{CH}_{3} \mathrm{OH}$ from these results toward B1 and B2 are provided in Figures 2 and 3, respectively, overlaid on observations and corrected for beam efficiency and a $12^{\prime \prime}$ source size. The $\mathrm{CH}_{3} \mathrm{OH}$ column densities and $\mathrm{H}_{2}$ densities found here are in relatively good agreement with previous observations of the source (Bachiller et al. 1995; Bachiller \& Pérez Guti'eerrez 1997; Sugimura et al. 2010). The derived values of $T_{\text {kin }}$ for the warmer component, which we ascribe to the shocks, agree well with previous measurements in B2 (Lefloch et al. 2012), while the upper range of our derived value in B1 falls at the lower edge of previous measurements.

The kinetic temperature of the second component $\left(T_{\text {kin }}=10 \mathrm{~K}\right)$ is significantly lower than previous measurements of both the shock and the outflow $\left(T_{\text {kin }} \sim 60-120 \mathrm{~K}\right)$, indicating that it is likely not probing the outflow as we had originally presumed. Instead, toward B1, this component is more likely related to the $g_{3}$ component described by Lefloch et al. (2012) as remnants from the gas in which the earlier B2 shock was formed, also offering a possible explanation for the component's presence toward B2. The $g_{3}$ component has been reported to be both cold $\left(T_{\text {kin }}=23 \mathrm{~K}\right)$ and extended (Mendoza et al. 2014; Lefloch et al. 2012) in qualitatively good agreement with our fit.

\section{RESULTS}

Despite deep searches, we find no conclusive evidence for $\mathrm{NH}_{2} \mathrm{OH}$ emission at $\lambda=3 \mathrm{~mm}$ or $\lambda=1.3 \mathrm{~mm}$ in either the CARMA or CSO data, respectively. To calculate appropriate upper limits for $\mathrm{NH}_{2} \mathrm{OH}$ in the both data sets, we assume that $\mathrm{NH}_{2} \mathrm{OH}$, if present in the gas phase, originates in the warm, shocked gas traced by the warm, dense $\mathrm{CH}_{3} \mathrm{OH}$ component. The rationale for this assumption is discussed in Section 7.

\section{1. $\mathrm{NH}_{2} \mathrm{OH}$ in CSO Data}

Near the temperatures derived from our RADEX fit in the warm component toward $\mathrm{B} 1$ and $\mathrm{B} 2$, the strongest $\mathrm{NH}_{2} \mathrm{OH}$ transition is the $5_{0,5}-4_{0,4}$ at $251,838 \mathrm{MHz}$. As noted in Table 1, the critical density $\left(n_{\mathrm{cr}}\right)$ for this transition is $10^{5} \mathrm{~cm}^{-3}$. This value is based on the assumption that the collisional coefficients for $\mathrm{NH}_{2} \mathrm{OH}$ are similar to those of $A-\mathrm{CH}_{3} \mathrm{OH}$. While not exact, the similar mass, dipole moments, molecular size, and energy level structures make this a reasonable approximation within the context of the following discussion.

Given the densities derived from our RADEX fit (3-6 $\times 10^{5} \mathrm{~cm}^{-3}$ ), we therefore assume that, at least for this transition, LTE is a reasonable approximation for determining $\mathrm{NH}_{2} \mathrm{OH}$ upper limits, and thus $T_{\mathrm{ex}}=T_{\text {kin. }}$. Under these conditions, we derive $1 \sigma$ upper limit column densities for $\mathrm{NH}_{2} \mathrm{OH}$ of $\leqslant 1.4 \times 10^{14} \mathrm{~cm}^{-2}$ and $\leqslant 1.0 \times 10^{14} \mathrm{~cm}^{-2}$ in B1 and $\mathrm{B} 2$, respectively.

Lefloch et al. (2012) derive a CO column density of $0.9 \times 10^{17} \mathrm{~cm}^{-2}$ in the $g_{2}$ component of the B1 shock, arising from the shocked gas and covering the entire region assuming a $20^{\prime \prime}$ source size. Assuming that the $\mathrm{CO}$ is homogeneously distributed over the region, this gives a source-averaged $\mathrm{CO}$ column of $3.2 \times 10^{16} \mathrm{~cm}^{-2}$ for the $12^{\prime \prime}$ source size used here. Taking $N_{\mathrm{H}_{2}}=3 \times 10^{4}\left(N_{\mathrm{CO}}\right)$ (see Lefloch et al. 2012; Bolatto et al. 2013), this results in an $\mathrm{H}_{2}$ column density of $\sim 10^{21} \mathrm{~cm}^{-2}$. A similar argument using the $\mathrm{CO}$ column density of $5.2 \times 10^{16} \mathrm{~cm}^{-2}$ for B2 found by Bachiller \& Pérez Guti'eerrez (1997) also results in an $\mathrm{H}_{2}$ column density of $\sim 10^{21} \mathrm{~cm}^{-2}$. This gives upper limits to the fractional abundances of $\mathrm{NH}_{2} \mathrm{OH}$ of $N_{\mathrm{NH}_{2} \mathrm{OH}} / N_{\mathrm{H}_{2}}=\leqslant 1.4 \times 10^{-7}$ and $\leqslant 1.0 \times 10^{-7}$ for $\mathrm{B} 1$ and $\mathrm{B} 2$, respectively.

\section{2. $\mathrm{NH}_{2} \mathrm{OH}$ in CARMA Data}

To calculate appropriate upper limits for $\mathrm{NH}_{2} \mathrm{OH}$ in the CARMA data, we again assume that $\mathrm{NH}_{2} \mathrm{OH}$, if present in the gas phase, originates in the warm, shocked gas traced by the warm, dense $\mathrm{CH}_{3} \mathrm{OH}$ component. The spectra extracted from a $12^{\prime \prime}$ beam centered on these locations are shown in Figure 4. While there is an unidentified feature at the frequency of the $2_{1,2}-1_{1,1} \quad \mathrm{NH}_{2} \mathrm{OH}$ transition, if this were truly $\mathrm{NH}_{2} \mathrm{OH}$ emission, then the other transitions in the observed window would be of equal or greater intensity.

We measure the rms to be 1.6 and $2.9 \mathrm{mK}$ in the B1 and B2 spectra. At the derived temperatures, the $2_{0,2}-1_{0,1}$ transitions at $100,748 \mathrm{MHz}$ are predicted to be strongest. As both B1 and B2 have densities higher than $n_{\mathrm{cr}}$ for this transition (see Table 1), we again derive an upper limit to the column density assuming LTE conditions.

Following the same procedure as for the CSO data, we find a $1 \sigma$ upper limit of $\mathrm{NH}_{2} \mathrm{OH}$ of $\leqslant 1.4 \times 10^{13} \mathrm{~cm}^{-2}$ for $\mathrm{B} 1$ and $\leqslant 1.5 \times 10^{13} \mathrm{~cm}^{-2}$ for $\mathrm{B} 2$. This gives upper limits to the fractional abundances of $\mathrm{NH}_{2} \mathrm{OH}$ of $N_{\mathrm{NH}_{2} \mathrm{OH}} / N_{\mathrm{H}_{2}}=$ $\leqslant 1.4 \times 10^{-8}$ and $\leqslant 1.5 \times 10^{-8}$ for $\mathrm{B} 1$ and $\mathrm{B} 2$, respectively. All of our results are summarized in Table 4 .

\section{DISCUSSION AND CONCLUSIONS}

In Section 6, we assumed that $\mathrm{NH}_{2} \mathrm{OH}$, if present, would arise predominantly from the warm, shocked regions B1 or B2, rather than the molecular outflow. Zheng \& Kaiser (2010) show that $\mathrm{NH}_{2} \mathrm{OH}$ is thermally liberated from their laboratory samples between 160-180 K, whereas for realistic interstellar ices and conditions, temperatures above $\sim 110 \mathrm{~K}$ are likely sufficient (Collings et al. 2004). Both our non-LTE fits, and the literature values discussed earlier have shown that temperatures in the targeted regions are below these thresholds, and thus a thermal mechanism for the desorption of $\mathrm{NH}_{2} \mathrm{OH}$ from grain surfaces in L1157 is unlikely. Thus, non-thermal desorption in the shocks should be the dominant mechanism for $\mathrm{NH}_{2} \mathrm{OH}$ 

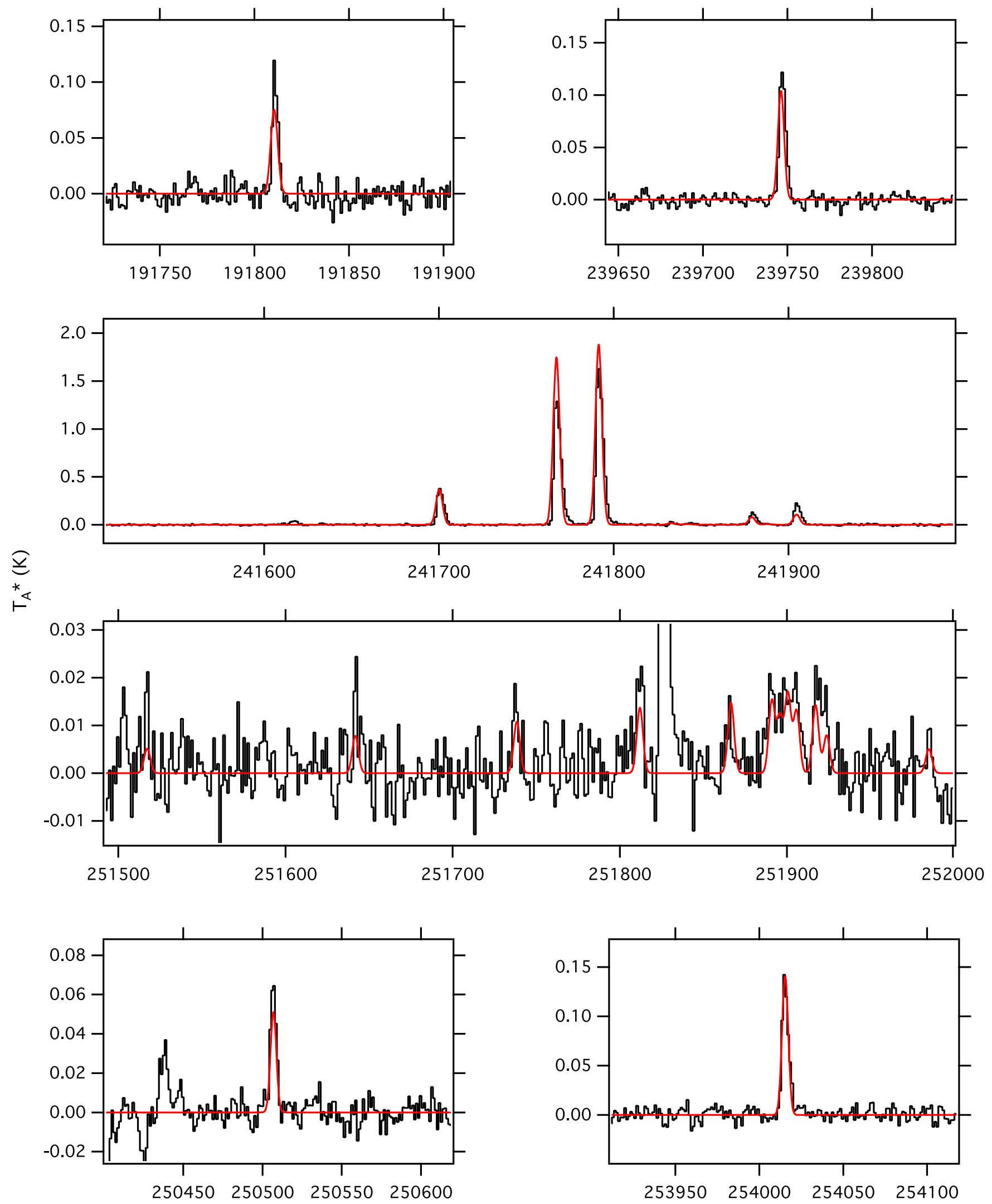

Frequency $(\mathrm{MHz})$

Figure 2. Non-LTE simulation of $\mathrm{CH}_{3} \mathrm{OH}$ spectrum from RADEX fit in red, overlaid on CSO observations of the B1 shock in black. The $4_{1,4}-3_{1,3}{ }^{++}$transition of $A$ $\mathrm{CH}_{3} \mathrm{OH}$ at $191810.5 \mathrm{MHz}$ was not included as part of the RADEX fit. It is, however, reasonably well reproduced by the fit of the higher-frequency lines, adding additional evidence for the robustness of the fit. 

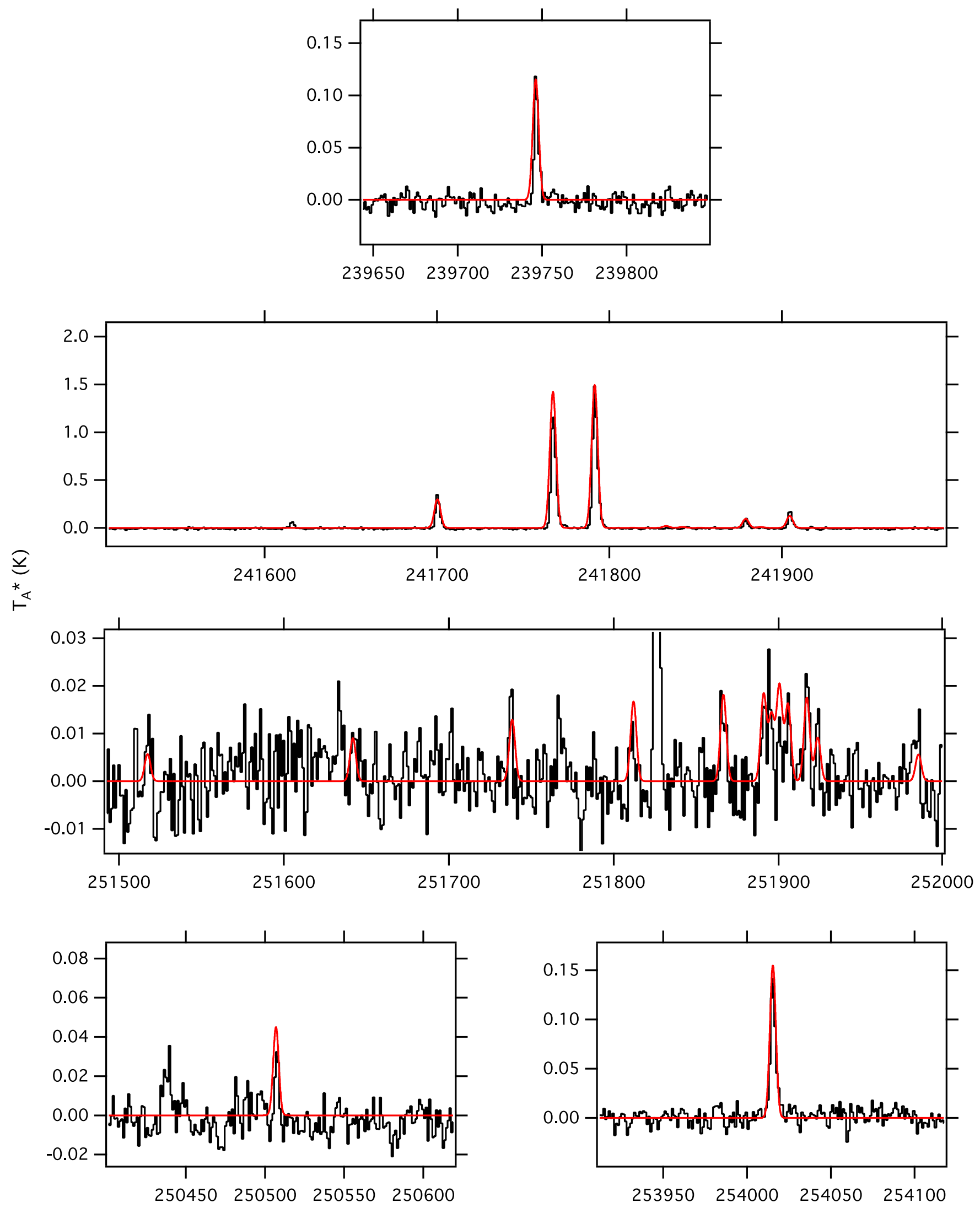

Frequency $(\mathrm{MHz})$

Figure 3. Non-LTE simulation of $\mathrm{CH}_{3} \mathrm{OH}$ spectrum from RADEX fit shown in red, overlaid on CSO observations of the B2 shock in black. 


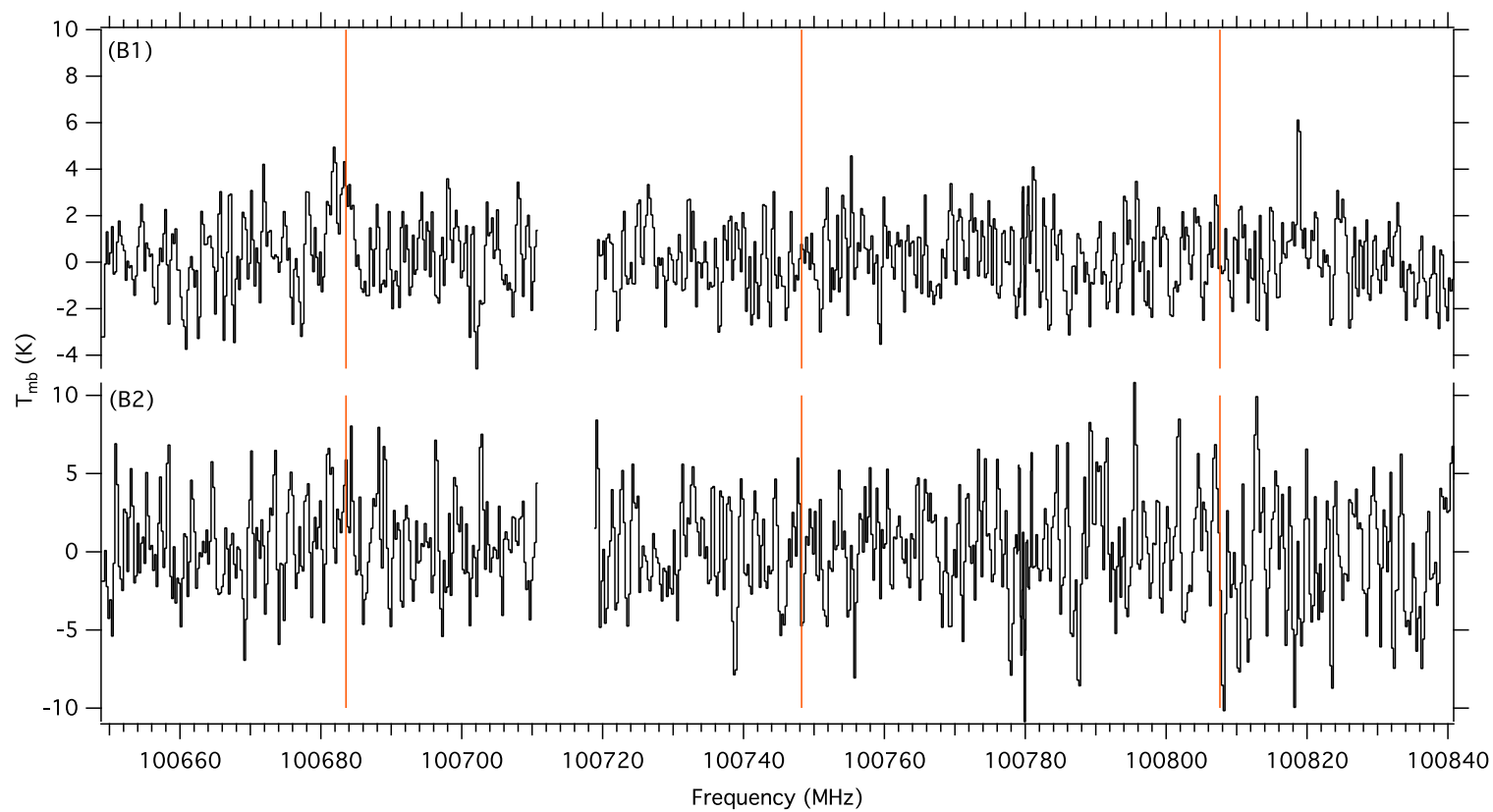

Figure 4. Spectra toward L1157-B1 (bottom) and B2 (top) extracted from 12" beams centered on the peaks of the shocked $\mathrm{CH}_{3} \mathrm{OH}$ emission and smoothed to a velocity resolution of $\sim 1.4 \mathrm{~km} \mathrm{~s}^{-1}$. Red lines indicate the frequencies of $\mathrm{NH}_{2} \mathrm{OH}$ transitions in this frequency region. A U line is present toward the $\mathrm{B} 1$ shock at the frequency of the $2_{1,2}-1_{1,1} \mathrm{NH}_{2} \mathrm{OH}$ transition.

Table 4

Derived Upper Limits to $\mathrm{NH}_{2} \mathrm{OH}$ Column Density and Relative Abundance in L1157-B1 and L1157-B2 from the CSO and CARMA Data

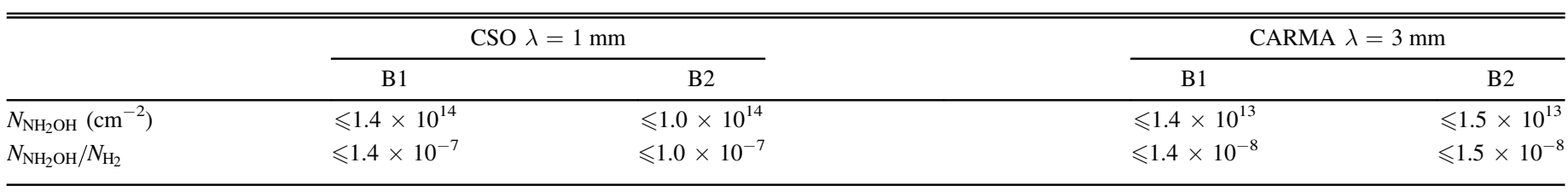

liberation, and any $\mathrm{NH}_{2} \mathrm{OH}$ emission should trace these shocked regions.

The upper limit column densities established with the CARMA data are equivalent to those established by Pulliam et al. (2012) of $\leqslant(0.9-8) \times 10^{13} \mathrm{~cm}^{-2}$. The limits established by Pulliam et al. (2012), however, are beam-averaged column densities, whereas the CARMA measurements presented here provide a more well-constrained source size, and thus a more robust upper limit. Nevertheless, the upper limits to relative abundance found here are significantly higher than those determined by Pulliam et al. (2012) of $\sim 10^{-10}$ due to the low $\mathrm{H}_{2}$ column in the region. These low columns are common in other chemically-complex shocked regions as well: the host of Galactic Center clouds studied by Requena-Torres et al. (2006; $\left.N_{\mathrm{H}_{2}}=(2-68) \times 10^{21} \mathrm{~cm}^{-2}\right)$ and other shocked outflows like BHR $71\left(N_{\mathrm{H}_{2}}=(3-11) \times 10^{21} \mathrm{~cm}^{-2}\right.$; Garay et al. 1998).

Yet, these regions likely represent the best-case scenario for a gas-phase detection of $\mathrm{NH}_{2} \mathrm{OH}$. Such a detection is critical to accurately constrain the application of laboratory results, which show that $\mathrm{NH}_{2} \mathrm{OH}$ is a significant player in grain-surface nitrogen chemistry to chemical models. The deep search presented here stretched the capabilities of the CSO and CARMA to the limit; searches deep enough to be sensitive to the relative abundances predicted by laboratory work and modeling are simply time-prohibitive on these types of instruments. The Garrod (2013) model, without the addition of the $\mathrm{H}+\mathrm{HNO} \rightarrow \mathrm{HNOH}$ pathway, predicts peak condensedphase $\mathrm{NH}_{2} \mathrm{OH}$ abundances of $\sim(5-10) \times 10^{-9}$, which, if liberated en masse in a shock, is a detectable population, but likely only with the sensitivity and spatial resolution of ALMA. The upper limits established here, however, are several orders of magnitude lower than both the condensed-phase and gasphase populations predicted by the augmented model which includes this hydrogenation pathway. Thus, our results demonstrate that further efforts are needed both in modeling and in the laboratory, to identify and fully constrain both the formation and the destruction pathways for $\mathrm{NH}_{2} \mathrm{OH}$.

B.A.M. gratefully acknowledges funding by an NSF Graduate Research Fellowship during the initial portions of this work. The authors thank the anonymous referee for comments that increased the quality of this work. We thank S. Radford for support of the CSO observations. The National Radio Astronomy Observatory is a facility of the National Science Foundation operated under cooperative agreement by Associated Universities, Inc. Portions of this material are based upon work at the Caltech Submillimeter Observatory, which was operated by the California Institute of Technology under cooperative agreement with the National Science Foundation (AST-0838261). Support for CARMA construction was derived from the Gordon and Betty Moore Foundation, the Kenneth T. and Eileen L. Norris Foundation, the James S. McDonnell Foundation, the Associates of the California Institute of Technology, the University of Chicago, the states of California, Illinois, and Maryland, and the National Science Foundation. 


\section{APPENDIX \\ ERROR ANALYSIS}

The uncertainty in the observations is given as Equation (1), modified from Equation (A1) in Crockett et al. (2014):

$$
\sigma_{\mathrm{tot}}=\sqrt{\sigma_{\mathrm{rms}}^{2}+\sigma_{\mathrm{cal}}^{2}+\sigma_{\mathrm{pt}}^{2}+\sigma_{\mathrm{bf}}^{2}} .
$$

$\sigma_{\text {rms }}$ is the uncertainty due to the rms noise level of the spectra. This value is consistently $\sim 0.0058 \mathrm{~K}$ across the $\mathrm{CH}_{3} \mathrm{OH}$ transitions in $\mathrm{B} 1$, and $\sim 0.0078 \mathrm{~K}$ in $\mathrm{B} 2$.

$\sigma_{\text {cal }}$ is the uncertainty in the absolute flux calibration of the observations, which is $\sim 30 \%$.

$\sigma_{\mathrm{pt}}$ is the uncertainty in the pointing accuracy, which is taken to be $2^{\prime \prime}$ based on the average convergence of pointing observations throughout the observing period.

$\sigma_{\mathrm{bf}}$ is the uncertainty in the beam-filling factor. The size of the telescope beam varies by $\sim 4^{\prime \prime}$ across the band, is well described, and is calculated independently at each transition frequency, and thus we assume no contribution to $\sigma_{\mathrm{bf}}$ from the telescope beam. However, the sizes of the two source components are not a varied parameter, and are only modestly well-described by our CARMA observations. Furthermore, in the case of B1, the shock is positioned off-center of the beam, making the emission more sensitive to fall-off effects that are not accounted for explicitly in the calculations. We therefore assume a factor of two uncertainty in source size to take both issues into consideration, which is reflected in $\sigma_{\mathrm{bf}}$.

These result in net uncertainties of $\sim 32 \%$ in the absolute intensity of the observed transitions, with the largest contribution coming from the absolute flux calibration. $\chi^{2}$ values outside of the extremes represented by these uncertainties more than double those of the best-fit parameters, and so we take $\sim 32 \%$ to be a reasonable estimate of the overall uncertainty in $n_{\mathrm{H}_{2}}, N_{\mathrm{CH}_{3} \mathrm{OH}}$, and $T_{\text {kin }}$.

\section{REFERENCES}

Angelelli, F., Aschi, M., Cacace, F., Pepi, F., \& de Petris, G. 1995, JPhCh, 99,6551

Arce, H. G., Santiago-García, J., Jørgensen, J. K., Tafalla, M., \& Bachiller, R. 2008, ApJL, 681, L21

Bachiller, R., Liechti, S., Walmsley, C. M., \& Colomer, F. 1995, A\&A, 295, L51

Bachiller, R., Martín-Pintado, J., \& Fuente, A. 1993, ApJL, 417, L45

Bachiller, R., \& Pérez Guti'eerrez, M. 1997, ApJL, 487, L93

Barrientos, C., Redondo, P., Largo, L., Rayon, V. M., \& Largo, A. 2012, ApJ, 748, 99

Blagojevic, V., Petrie, S., \& Bohme, D. K. 2003, MNRAS, 339, L7
Bolatto, A. D., Wolfire, M., \& Leroy, A. K. 2013, ARA\&A, 51, 207

Boulet, P., Gilardoni, F., Weber, J., Chermette, H., \& Ellinger, E. 1999, CP, 244, 163

Codella, C., Fontani, F., Ceccarelli, C., et al. 2015, MNRAS, 449, L11

Collings, M. P., Anderson, M. A., Chen, R., et al. 2004, MNRAS, 354, 1133

Congiu, E., Chaabouni, H., Laffon, C., et al. 2012b, JChPh, 137, 054713

Congiu, E., Fedoseev, G., Ioppolo, S., et al. 2012a, ApJL, 750, L12

Crockett, N. R., Bergin, E. A., Neill, J. L., et al. 2014, ApJ, 787, 112

Cunningham, M. R., Jones, P. A., Godfrey, P. D., et al. 2007, MNRAS, 376, 1201

Elsila, J. E., Glavin, D. P., \& Dworkin, J. P. 2009, M\&PS, 44, 1323

Fedoseev, G., Ioppolo, S., Lamberts, T., et al. 2012, JChPh, 137, 054714

Garay, G., Köhnenkamp, I., Bourke, T. L., Rodríguez, L. F., \& Lehtinen, K. K. 1998, ApJ, 509, 768

Garrod, R. T. 2013, ApJ, 765, 60

Garrod, R. T., Widicus Weaver, S. L., \& Herbst, E. 2008, ApJ, 682, 283

Gueth, F., Guilloteau, S., \& Bachiller, R. 1996, A\&A, 307, 891

He, J., Vidali, G., Lemaire, J.-L., \& Garrod, R. T. 2015, ApJ, 799, 49

Ioppolo, S., Fedoseev, G., Minissale, M., et al. 2014, PCCP, 16, 8270

Jones, P. A., Cunningham, M. R., Godfrey, P. D., \& Cragg, D. M. 2007, MNRAS, 374, 579

Kuan, Y.-J., Charnley, S. B., Huang, H.-C., Tseng, W.-L., \& Kisiel, Z. 2003, ApJ, 593, 848

Kvenvolden, K., Lawless, J., Pering, K., et al. 1970, Natur, 228, 923

Largo, L., Rayón, V. M., Barrientos, C., Largo, A., \& Redondo, P. 2009, CPL, 476, 174

Lefloch, B., Cabrit, S., Busquet, G., et al. 2012, ApJL, 757, L25

Looney, L. W., Tobin, J. J., \& Kwon, W. 2007, ApJL, 670, L131

Mehringer, D. M., Snyder, L. E., Miao, Y., \& Lovas, F. J. 1997, ApJL, 480, L71

Mendoza, E., Lofloch, B., López-Sepulcre, A., et al. 2014, MNRAS, 445, 151

Minissale, M., Fedoseev, G., Congiu, E., et al. 2014, PCCP, 16, 8257

Morino, I., Yamada, K. M. T., Klein, H., et al. 2000, JMoST, 517, 367

Müller, H. S. P., Menten, K., \& Mäder, H. 2004, A\&A, 428, 1019

Müller, H. S. P., Schlöder, F., Stutzki, J., \& Winnewisser, G. 2005, JMoST, 742,215

Pulliam, R. L., McGuire, B. A., \& Remijan, A. J. 2012, ApJ, 751, 1

Rabli, D., \& Flower, D. R. 2010, MNRAS, 406, 95

Requena-Torres, M. A., Martín-Pintado, J., Rodríguez-Franco, A., et al. 2006, A\&A, 455, 971

Schöier, F. L., van der Tak, F. F. S., van Dishoeck, E. F., \& Black, J. H. 2005, A\&A, 432, 369

Snow, J. L., Orlova, G., Blagojevic, V., \& Bohme, D. K. 2007, JACS, 129, 9910

Snyder, L. E., Lovas, F. J., Hollis, J. M., et al. 2005, ApJ, 619, 914

Sugimura, M., Takahiro, Y., Takeshi, S., et al. 2010, PASJ, 63, 459

Tafalla, M., \& Bachiller, R. 1995, ApJL, 443, L37

Tsunekawa, J. 1972, JPSJ, 33, 167

van der Tak, F. F. S., Black, J. H., Schöier, F. L., Jansen, D. J., \& van Dischoeck, E. F. 2007, A\&A, 468, 627

van Dishoeck, E. F., \& Blake, G. A. 1998, ARA\&A, 36, 317

Xu, L.-H., \& Lovas, F. J. 1997, JPCRD, 26, 17

Zheng, W., \& Kaiser, R. I. 2010, JPCA, 114, 5251 